\title{
Specific Impedance of Cerebral Cortex during Spreading Depression, and an Analysis of Neuronal, Neuroglial, and Interstitial Contributions
}

\author{
JAMES B. RANCK, JR. ${ }^{1}$ \\ Department of Physiology, University of Michigan, Ann Arbor, Michigan
}

Received July 23, 1963

The specific impedance of cerebral cortex of rabbits anesthetized with urethane was measured during spreading cortical depression (SD) at frequencies from 5 to $50,000 \mathrm{cycle} / \mathrm{sec}$. During SD the amplitude of impedance increased at all frequencies, the maximum occurring progressively later at lower frequencies for those less than 5,000 cycle/sec. The phase angle increased at 5,000 and 50,000 cycle/sec with the same time course as the changes in amplitude of impedance. At $50 \mathrm{cycle} / \mathrm{sec}$ the phase angle initially decreased, sometimes to zero, and then increased to greater than the pre-SD state, with the maximum occurring later than the maximum in amplitude of impedance at this frequency. At $500 \mathrm{cycle} / \mathrm{sec}$ the phase angle initially decreased or did not change. It then increased, the maximum occurring later than the maximum of the amplitude of impedance at 500 cycle $/ \mathrm{sec}$, but earlier than the maximum phase angle at $50 \mathrm{cycle} / \mathrm{sec}$. These data are interpreted in terms of a previously published analysis of specific impedance of cerebral cortex. Three processes must occur during SD. Early in SD the size of the interstitial space of cortex must decrease and the membrane resistance of neurons must decrease. Later the membrane resistance of neuroglia must increase. The significance of these processes is discussed with relevance to SD and cortical processes in general. It is suggested that the membrane change of neuroglia is similar to that of "anomalous rectification" of skeletal muscle.

\section{Introduction}

In 1953 Lẽao and Martins Ferreira (13) reported the increase of specific impedance of cerebral cortex during spreading depression (SD), and this finding has been corroborated frequently since then $(14,16)$. However, only the amplitude of impedance has been measured and this only at about $1000 \mathrm{cycle} / \mathrm{sec}$.

1 This investigation was supported by grants NIH B-1068 and NIH NB-04352 from the National Institutes of Health. The author thanks Dorothy Luciano, Frances Bignall, and Grace Lasterie for their assistance. 
In a previous paper it was shown that the specific impedance of cerebral cortex of rabbit is frequency dependent (18). An analysis of this impedance data showed how impedance measurements can be used to analyze changes in membrane resistances of neurons and neuroglia and changes in the size of interstitial space (19). In this paper these impedance methods and analyses are applied to the changes during SD. Three different processes will be shown to occur during SD: (a) the membrane resistance of neurons decreases; (b) the size of interstitial space decreases; and (c) the membrane resistance of neuroglia increases.

\section{Methods}

Rabhits were anesthetized with urethane. The trachea was cannulated and the cannula attached to a positive-pressure respirator. A bilateral pneumothorax was then performed. One side of the cerebrum was widely exposed. The specific impedance of cerebral cortex was measured, using a method previously described (18). Briefly, sinusoidally varying current was passed from a $20-40 \mu$ platinum electrode, and the voltage difference was measured between two similar electrodes within $1.3 \mathrm{~mm}$ of the current electrode. The animal was grounded through a $470-\mathrm{ohm}$ current monitoring resistor, and the voltage across the resistor drove the horizontal sweep of a Tektronics 502 oscilloscope. The voltage difference between the two voltage electrodes on the cortical surface drove the vertical sweep.

Before and after each SD the effect of interelectrode capacities was checked by recording the voltage with the current electrode lifted off the surface of cortex. Results were used only at frequencies less than those at which any interelectrode capacity effects could be seen. The frequency response of the whole system was checked at the end of each day by running the experiment on a layer of $0.05 \mathrm{M} \mathrm{NaCl}$ agar more than $2 \mathrm{~mm}$ thick on the cortical surface. The frequency response on agar was always good to at least $50 \mathrm{kc} / \mathrm{sec}$; therefore, frequencies less than this are considered valid. However, the more stringent criteria used in a previous paper of accepting frequencies only less than one-fourth the value good in agar was seldom met.

Electroencephalograms were recorded between one of the voltage electrodes and a screw in the frontal sinus. No other characteristics of SD were measured, but, as will be shown below, the impedance changes in SD are so stereotyped that they alone can be used to identify SD. Spreading depressions were initiated by passing $200 \mu \mathrm{a}(\mathrm{rms})$ from the current elec- 
trode at $50 \mathrm{cycle} / \mathrm{sec}$ for about $1 \mathrm{sec}$. (Impedance measurements were made with about $5 \mu \mathrm{a}$ rms.) At least 15 min elapsed between SD.

Impedances were measured at 5, 50, 500, 5,000, and 50,000 cycle/sec. Photographs were taken of the oscilloscope face, and the time of each photograph was marked on an EEG record. Exposures were for $0.2 \mathrm{sec}$, so that only one cycle of 5 cycle/sec was photographed. This made it difficult to be sure of phase angles for the 5 cycle/sec frequency from photographs. However, the oscilloscope face was observed visually and many cycles were averaged by eye. Even though occasional suggestive phase angles at $5 \mathrm{cycle} / \mathrm{sec}$ were seen, these occurred less than six times in all records, and they were inconstant in relation to EEG, SD, and other characteristics of the impedance. Therefore phase angle at 5 cycle/sec will be assumed to be zero. Reasons showing that it would be unlikely for a nonzero phase angle to be present at $5 \mathrm{cycle} / \mathrm{sec}$ will be given below.

The impedance of cerebral cortex was measured from before an SD until after the FEG had returned to the pre-SD state. This lapse of time was at least $4 \mathrm{~min}$, and during it there was often drying of the cortex, which presumably changed the electrode to cortex resistances and therefore the effect of interelectrode capacities. The cortical swelling during SD might also change this effect of interelectrode capacities. To control for these effects, some experiments were run with the electrodes on the cortical surface in air, some with the electrodes piercing the pial surface with the cortex in air and some with the cortex under a layer of mineral oil. With the electrodes piercing the pia, the magnitude and phase angle of the specific impedance were always smaller than for the other cases, although the relative changes and the timing of changes of specific impedance were the same in all cases. When the electrodes pierced the pia more fluid seemed to collect around the electrode on the cortical surface than in the other cases. Piercing the pia may have changed the characteristics of the surrounding cortex. However, no attempt was made to investigate these factors.

In thirty SD taken on eight rabbits, specific impedance during SD was measured, rotating as rapidly as possible through the five frequencies used. Usually from four to six measurements of each frequency could be made in $1 \mathrm{~min}$. Spreading depressions were also observed measuring impedance constantly with a single frequency to show that no rapid fluctuations occur and that a smooth curve drawn through discrete points is justified. At least two SD were run in this way for each of the five standard frequencies. Six runs were made in which care was taken to eliminate fluid 
on the surface of the pia and to keep the electrodes away from large blood vessels. In these runs frequencies up to $50,000 \mathrm{cycle} / \mathrm{sec}$ were valid. The data from these six runs will always be used when quantitative data are given. There were no qualitative differences between changes in these six SD and others.

This method of measuring the specific impedance of cortex assumes hemispherical symmetry and measures the specific impedance of a shell of cortex. The SD was started from the current electrode at the center of the shell in which impedance is measured. There is evidence that SD involves superficial and deep layers of cortex differently and that a propagated wave of SD occurs in the superficial layers before it does in the deep layers (16). Therefore, the assumption of hemispherical symmetry may well not be correct during SD. However, all of the impedance changes observed are sufficiently large and all of the quantitative arguments hold when considered to be only order of magnitude arguments, so that the error of the assumption of hemispherical symmetry will not affect the conclusions. Isotropy is also assumed without clear evidence to justify it. However, there seems little reason to doubt it.

\section{Results}

The specific impedance of cerebral cortex without SD was the same as previously reported (18) except that the phase angle at $5,000 \mathrm{cycle} / \mathrm{sec}$ was often as large as 3-4 degrees and only rarely less than 1 degree. However, the phase angle at $5,000 \mathrm{cycle} / \mathrm{sec}$ in the non-SD was always about one-half to one-third that at $50 \mathrm{cycle} / \mathrm{sec}$ and always less than that at 500 cycle/sec. These data do not invalidate the previous analysis; however, some revisions are necessary and will be given in the discussion.

Figure 1 gives the change in specific impedance during SD. All differences from the pattern illustrated in Fig. 1 will be specifically stated below. Spreading depression was invariably associated with an increase in the amplitude of the specific impedance of cortex at all frequencies tested. The maximum of the amplitude of impedance $(|Z|)$ at $5 \mathrm{cycle} / \mathrm{sec}$ invariably occurred later than the maximum of $|\mathrm{Z}|$ at $50 \mathrm{cycle} / \mathrm{sec}$, which in turn was later than that for higher frequencies. The maximum of $|Z|$ at $500 \mathrm{cycle} / \mathrm{sec}$ sometimes had occurred before the maximum of $|Z|$ at higher frequencies and sometimes at the same time; $|\mathrm{Z}|$ always had the same time course at 5,000 and at 50,000 cycle/ $/ \mathrm{sec}$. The phase angle at 5,000 and 50,000 cycle/sec always increased and with the same time course as changes in $|Z|$ at these frequencies. The phase angle at $500 \mathrm{cycle} / \mathrm{sec}$ 
sometimes decreased at the beginning of SD for about $15 \mathrm{sec}$, but usually there was no change for about $15 \mathrm{sec}$. It then invariably increased to a value even greater than that in the pre-SD state; the maximum phase angle always occurred later than the maximum in $\left|Z_{1}\right|$ at $500 \mathrm{cycle} / \mathrm{sec}$. At

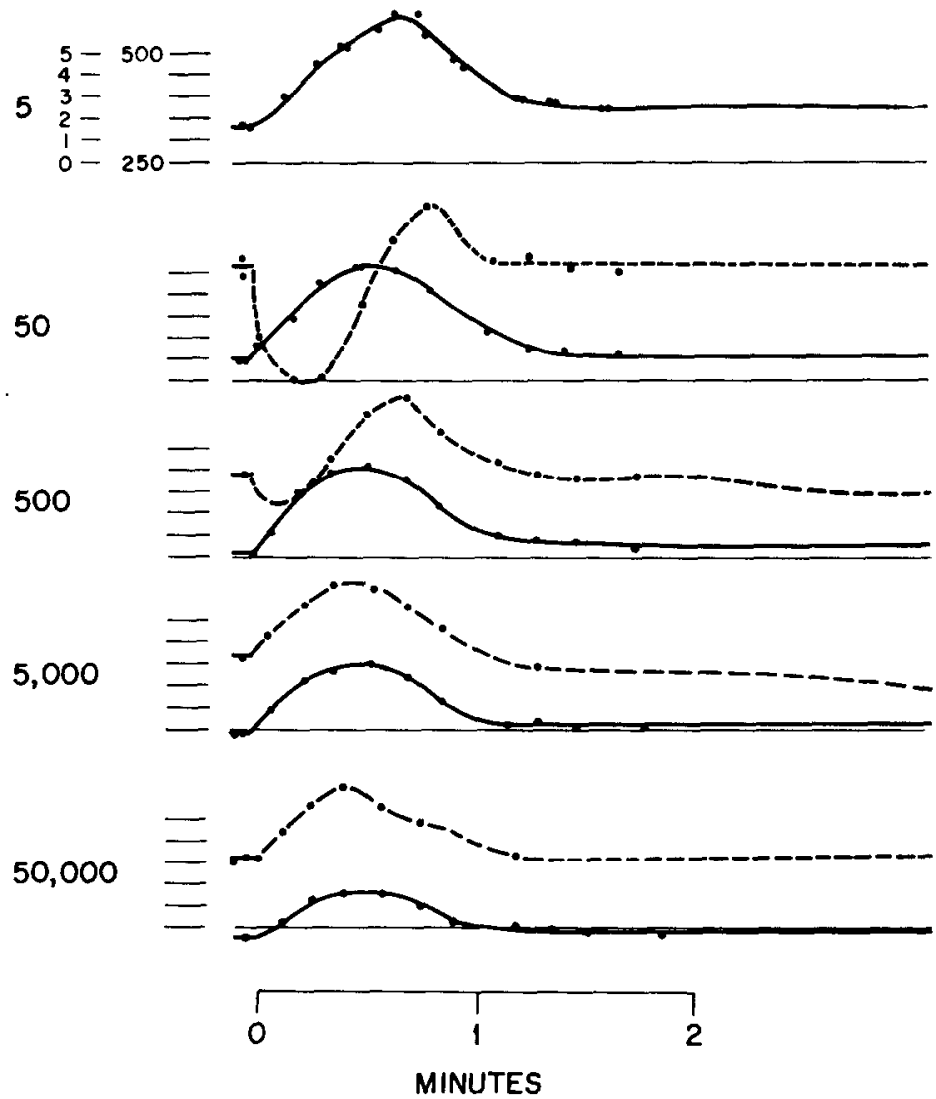

Fit. 1. Impedance changes at 5, 50, 500, 5,000 and 50,000 cycle/sec. The SD is initiated at zero minutes. The solid line is the amplitude of specific impedance. The dotted line is the phase angle of specific impedance. The calibration is phase angle in degrees on the leit, and amplitude in ohm-cm on the right. The dots are the experimental points. The line connecting the dots was drawn by eye.

$50 \mathrm{cycle} / \mathrm{sec}$ there was always an immediate decrease in phase angle, sometimes to zero, for about $30 \mathrm{sec}$; then the phase angle increased to greater than pre-SD state, the maximum always occurring after the maximum of $|Z|$ at $50 \mathrm{cycle} / \mathrm{sec}$. The maximum of the $50 \mathrm{cycle} / \mathrm{sec}$ phase angle 
was invariably later than that of the $500 \mathrm{cycle} / \mathrm{sec}$, which was later than that at 5,000 and $50,000 \mathrm{cycle} / \mathrm{sec}$.

When the data are plotted in polar form, as in Fig. 2, several other features emerge. In the first $10 \mathrm{sec}$ the frequency with the greatest phase angle goes from about $50 \mathrm{cycle} / \mathrm{sec}$ to about $500 \mathrm{cycle} / \mathrm{sec}$. Then gradu-

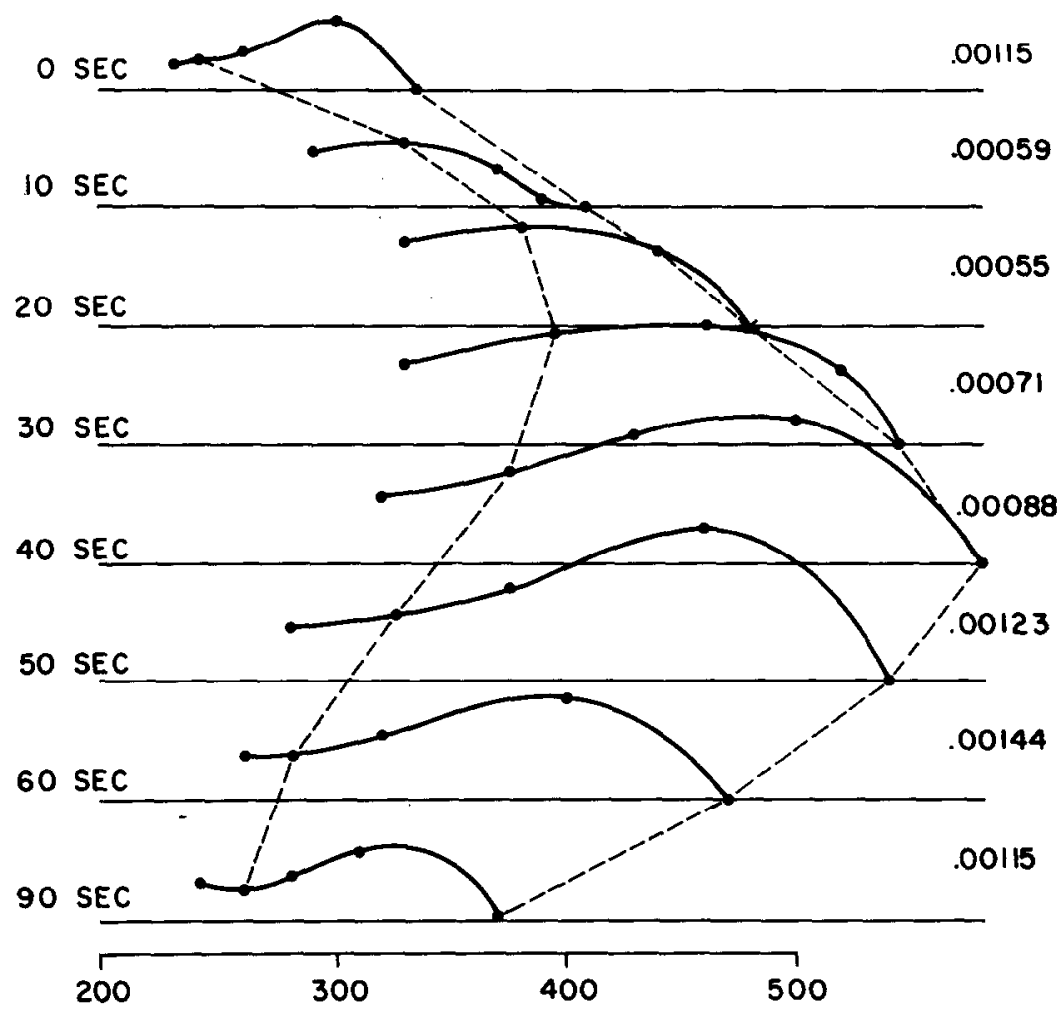

Fig. 2. The data from Fig. 1 are plotted here in polar form at the indicated times. These are eight separate polar plots. The origins of the plots is not shown, but would be off the diagram to the left. The dots are the measured values of impedance at 5, 50, 500, 5,000 and 50,000 cycle/sec. At $20 \mathrm{sec}$, the 5 and $50 \mathrm{cycle} / \mathrm{sec}$ values are the same. They are denoted by a cross. The continuous solid lines were drawn by eye. The dotted lines connect the $5 \mathrm{cycle} / \mathrm{sec}$ or $5,000 \mathrm{cycle} / \mathrm{sec}$ points in subsequent plots. The numbers to the right give the difference in conductance between $5 \mathrm{cycle} / \mathrm{sec}$ and $5,000 \mathrm{cycle} / \mathrm{sec}(\Delta Y)$ in $\mathrm{mho} / \mathrm{cm}$. The abscissa is the real part of the specific impedance and is labelled in ohm-cm. The ordinate is the imaginary part of the specific impedance and is to the same scale as the real part with negative up. 
ally the frequency of maximum phase angle decreases again to 50 cycle/ sec. The largest phase angle seen was always at $50 \mathrm{cycle} / \mathrm{sec}$.

\section{Interpretation of the Data in Terms of the Model}

At least three different processes must occur during SD to explain the impedance data: (a) the size of interstitial space must decrease; (b) membrane resistance of neurons must decrease; and (c) membrane resistance of neuroglia must increase.

(a) It has been shown in the analysis of the non-SD impedance data that at 5,000 and 50,000 cycle/sec current flow down the axis of fibers (longitudinal) is effectively at infinite frequency (19, pp. 164-165), and so it is independent of membrane resistance. It was argued that at these frequencies the transverse current flow is effectively at d-c $(19, \mathrm{pp}, 167-$ 168). Some modifications of this claim are given in the discussion. In any event, little transverse current flows through cells at these frequencies, and that which does is largely independent of membrane resistance for "reasonable" values of membrane resistance (19, p. 170). Since large changes in the sizes and shapes of cells do not occur in SD, the increases in the amplitude of the specific impedance at 5,000 and 50,000 cycle/sec must be due to an increase in cytoplasmic resistivity, an increase in the resistivity of interstitial space, a decrease in the size of interstitial space, or a decrease in the current carried by blood vessels. There are no known physical or biological reasons for thinking that major changes in cytoplasmic or interstitial resistivity could occur, so a decrease in the size of the interstitial space or vascular conductance is the only reasonable explanation of the data. Cortical blood vessels are dilated throughout most of SD so any change in vascular conductance would be an increase, not a decrease. Therefore, the increased amplitude of impedance at 5,000 and 50,000 $\mathrm{cycle} / \mathrm{sec}$ is due to a decrease in the size of the interstitial space. The changes in specific conductivity of these two frequencies always follow each other quite closely and one never varies from the other by more than $10 \%$. The decrease in specific conductivity ranged from 0.00075 to $0.00151 \mathrm{mho} / \mathrm{cm}$ with a mean of $0.00103 \mathrm{mho} / \mathrm{cm}$, so the interstitial space must normally contribute at least this much conductivity. An interstitial space of $10 \%$ of the total volume with a resistivity of $60 \mathrm{ohm}-\mathrm{cm}$ (an "equivalent conductive interstitial space" of $10 \%$ ) will contribute 0.00117 mho/cm of tissue conductivity, so normally the "equivalent conductive interstitial space" of brain must be at least $10 \%$. In a previous paper it 
was shown that the "equivalent conductive interstitial space" cannot be more than $15 \%$ (19).

The phase angle changes at 5,000 and 50,000 cycle/sec associated with SD do not have a direct explanation in terms of the model. However, as the size of the interstitial space decreases, a greater proportion of current can be expected to cross cell membranes, which would increase the phase angle of the impedance. (See Discussion for more on this point.) Since a solution for transverse current flow with very small interstitial space is not known, a quantitative fit to the data cannot be made.

(b) According to this model the difference in conductances between 5 and $5,000 \mathrm{cycle} / \mathrm{sec}$ is a measure of resistance to longitudinal current flow. Even without accepting the model it is clear that the difference in conductances of any two frequencies is at least monotonically related to some kind of an "average" membrane resistance. The difference between conductances at 5 and $5,000 \mathrm{cycle} / \mathrm{sec}$ always decreased during the first 10 sec of an SD to almost half of normal and this decrease always occurred with the same time course as the decrease in phase angle at $50 \mathrm{cycle} / \mathrm{sec}$. Therefore, there must initially be a decrease in the membrane resistance of that population which contributes to the impedance loop between 5 and 5,000 cycle/sec in the non-SD state. Neuronal membrane resistance must decrease.

The change in average neuronal membrane resistance can be determined if it is assumed that the neuronal population is homogeneous and neuroglia are not affecting the early change in impedance. The difference between the conductances at 5 and $5,000 \mathrm{cycle} / \mathrm{sec}(\Delta V)$ is ${ }^{2}$

$$
\left(p_{n} / 3 R_{c_{n}}\right)\left(2 \lambda_{n} / b_{n}\right) \tanh \left(b_{n} / 2 \lambda_{n}\right)
$$

where $p_{n}$ is the portion of tissue volume which is neuronal, $R_{c_{n}}$ is the resistivity of neuronal cytoplasm, $b_{n}$ is the distance across the dendritic arborization of neurons, and $\lambda_{n}$ is the average length constant of neuronal fibers which is equal to $\left(a_{n} R_{m_{n}} / 2 R_{c_{n}}\right)^{1 / 2}$ where $a_{n}$ is the average radius of neuronal fibers and $R_{m_{n}}$ is the specific membrane resistance of neurons. Assuming that only $R_{m_{n}}$ changes, the ratio of the minimum value of $\Delta Y\left(\Delta Y^{\prime}\right)$ to the pre-SD value of $\Delta Y\left(\Delta V^{\prime \prime}\right)$

$$
\begin{aligned}
& \Delta Y^{\prime} / \Delta Y^{\prime \prime}=\lambda_{n}{ }^{\prime} / \lambda_{n}{ }^{\prime \prime} \tanh (b / 2 \lambda)_{n} / \tanh \left(b / 2 \lambda^{\prime \prime}\right)_{n} \\
&=\left(R_{m_{n}}{ }^{\prime} / R_{m_{n}}{ }^{\prime \prime}\right)^{1 / 2} \tanh \left(b / 2 \lambda^{\prime}\right)_{\mathrm{n}} / \tanh \left(b / 2 \lambda^{\prime \prime}\right)_{n},
\end{aligned}
$$

where the superscripted primes and double primes denote the minimum

2 See reference 19, Eq. (11). 
and pre-SD values, respectively. $\Delta Y^{\prime} / \Delta Y^{\prime \prime}$ varies from 0.805 to 0.367 with a mean of 0.564 . Let $\left(2 \lambda^{\prime \prime} / b\right)_{n}=1.22$ as used previously (19). Let $R_{m_{n}}{ }^{\prime} / R_{m_{n}}{ }^{\prime \prime}=A .{ }^{2}$ Then $0.564=A \tanh (1 / 1.22 \mathrm{~A}) / \tanh (1 / 1.22)$. Solving by successive approximations, $R_{m_{n}}{ }^{\prime} / R_{m_{n}}{ }^{\prime \prime}=1 / 6.6$.

In Fig. 3 the line corresponding to impedance at $50 \mathrm{cycle} / \mathrm{sec}$ with only $R_{m}$ changing would, for the case of neurons, be close to the heavy lines

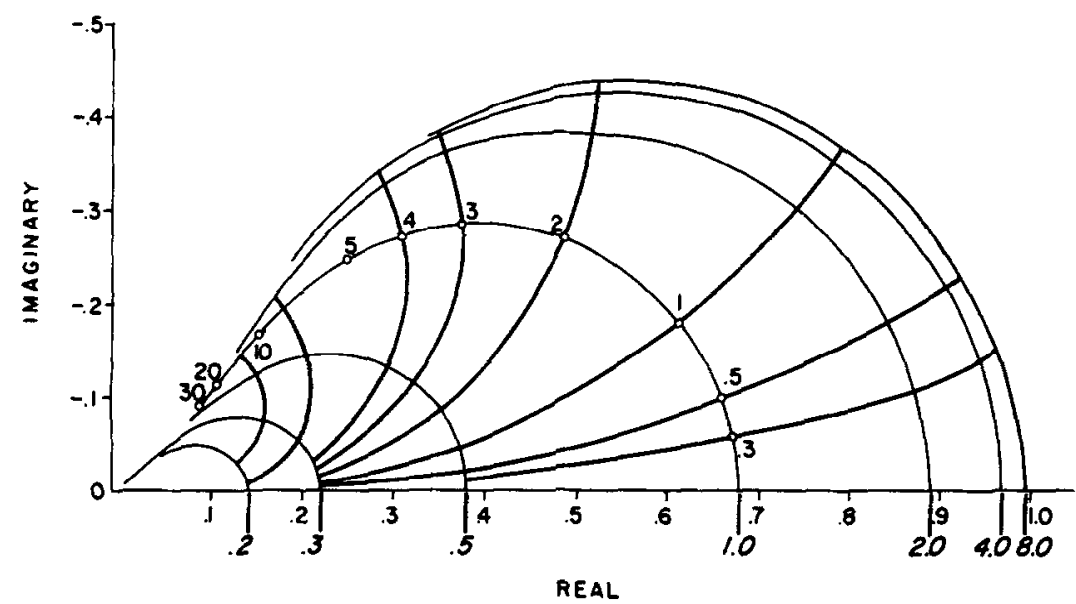

FIE. 3. The narrow lines are plots of normalized impedance loci which is the expression $Q$, defined in an earlier paper, with $p_{n} /\left(3 R_{c_{n}} Y_{b}\right)=0.5(19, \mathrm{p} .163$, Fig. 2). The numbers below the labels for the abscissa are the values of $2 \lambda / b$ for each impedance locus. The numbers on the $2 \lambda / b=1.0 \mathrm{plot}$ are values of $r \omega$. The heavy lines are plots of impedance at a single frequency with only $R_{m}$ and $2 \lambda / b$ become large without limit, the impedance locus becomes a semicircle with its center at 0.5 on the real axis. The constant frequency lines continue in the same direction as in the figure. Therefore the $2 \lambda / b=8.0$ locus is close to the limiting case.

passing through $\tau \omega=2.0$ or 3.0 on the $2 \lambda / b=1.0$ locus (19). $\omega$ is the frequency in radians per second and $\tau$ is the membrane time constant and is equal to $R_{m} C_{m}$, where $C_{m}$ is the specific membrane capacity in farads per $\mathrm{cm}^{2}$. The $500 \mathrm{cycle} / \mathrm{sec}$ line would pass through the $\tau \omega=20$ or 30 points on the $2 \lambda / b=1.0$ locus, although these lines are not drawn in Fig. 3. A four- to tenfold decrease in $R_{m_{n}}$ would move the impedance locus to $2 \lambda / b=0.3$ to 0.5 , which would be associated with a much larger decrease in phase angle at $50 \mathrm{cycle} / \mathrm{sec}$ than at $500 \mathrm{cycle} / \mathrm{sec}$. This was the experimental observation. The term $p_{n} /\left(3 R_{i_{n}} Y_{b}\right)$ has been held fixed at 0.5 in Fig. 3 , while in fact it will increase. However, even for $p_{n} /\left(3 R_{c_{n}} Y_{n}\right)$ 
$=2.0$, frequencies of maximum phase shift will change by no more than $20 \%$, and the shape of the impedance loci will change only negligibly.

In all of the analyses of changes in membrane resistance of neurons, there has been the tacit assumption that the same changes occurred simultaneously in all neurons, an assumption which in general is not justified. However, certain limits of the variability within the neuronal population can be determined. Since the 50-cycle phase angle goes to almost zero, it can be seen by following the $50 \mathrm{cycle} / \mathrm{sec}$ line on Fig. 3 that the value of $2 \lambda / b$ for most neurons must become less than 0.5 . Since initially the 500cycle phase angle sometimes did not measurably decrease at all, i.e., changed less than $1^{\circ}$ or about one-fourth of the pre-SD value, few of the neurons can have a value of $2 \lambda / b$ much less than 0.3 . Therefore the neurons must be acting fairly homogeneously.

As will be shown below, there is no evidence that the membrane resistance of neuroglia has begun to increase until after the time for which the minimum of neuronal membrane resistance has been calculated. Therefore the assumptions necessary to determine the quantitative changes in neuronal membrane resistance are approximately met up to that time. After that time it is not possible to quantitatively determine the neuronal membrane changes since the neuroglia are also changing. However, it will be shown below that the neuronal membrane resistance cannot have returned to the pre-SD value before the maximum phase angle occurs at $50 \mathrm{cycle} / \mathrm{sec}$.

(c) Starting at about $15 \mathrm{sec}$, the phase angle at 50 and $500 \mathrm{cycle} / \mathrm{sec}$ begins to increase, and associated with this increase is an increase in $\Delta Y$ which always increases at least to its pre-SD value and sometimes to as much as $25 \%$ greater than its pre-SD value. The membrane resistance of some population of cells must have increased. If the population of cells with the increased membrane resistance were the same population as that which initially decreased its membrane resistance, and if this population behaved homogeneously, then the time course of the change of amplitude of impedance would be the same at 5,50 and $500 \mathrm{cycle} / \mathrm{sec}$ and the time course of the phase angle would be the same at 50 and $500 \mathrm{cycle} / \mathrm{sec}$. This is not the case.

From Fig. 3 it can be seen that in order for the phase angle at 500 cycle/sec to increase earlier and more rapidly than at $50 \mathrm{cycle} / \mathrm{sec}$, the population of cells with increasing membrane resistance must have $2 \lambda / b$ less than about 0.3 if the constant frequency lines for 50 and $500 \mathrm{cycle} / \mathrm{sec}$ are the same as used above. But there cannot be much of the neuronal 
population with $2 \lambda / b$ less than 0.3 bccause of the restrictions on homogeneity discussed above. This increase cannot be due to neurons.

The value of $2 \lambda / b$ for neuroglia is less than about 0.1 in the non-SD state $(7,19)$. However, before an attempt can be made to fit the data with an increased membrane resistance of neuroglia, the constant frequency lines for neuroglia on the normalized impedance plot must be determined. The lumped term $p_{n} /\left(3 R_{c_{n}} Y_{b}\right)$ now becomes $p_{g} /\left(3 R_{c_{g}} Y_{b}\right)$ where $Y_{b}$ is now the total non-neuroglial admittance at $\mathrm{d}$-c. This lumped term will be about equal to 1.0 , so the loci will differ only slightly from those given in Fig. 3. For $(2 \lambda / b)_{n}=(2 \lambda / b)_{g}$, i.e., for loci with the same lumped parameter and $R_{c_{n}}=R_{c_{g}}, R_{m_{n}} / R_{m_{g}}=a_{g} / a_{n}\left(b_{n} / b_{g}\right)^{2}$ where the subscript $n$ refers to the neuronal population and $g$ to the neuroglial. As an example, assume $b_{g}=150 \mu, b_{n}=400 \mu, a_{g}=0.3 \mu, a_{n}=$ $0.4 \mu$ as used previously. Then $R_{m_{n}} / R_{n_{g}}=5$. While these values can only be guessed, it seems likely that the frequency for maximum phase shift at a given $2 \lambda / b$ will be somewhat higher for neuroglia than for neurons (a given frequency line is shifted clockwise on the normalized impedance plot for neuroglia $)$. The fact that $p_{g} /\left(3 R_{c_{g}} Y_{b}\right)$ is larger than the comparable term for neurons will also increase this effect. From the fact that the membrane resistance of neuroglia starts to increase from an initially lower value than neurons, and also from the clockwise shift of each frequency from neurons to neuroglia, an increase in the membrane resistance of neuroglia would increase the phase angle at $500 \mathrm{cycle} / \mathrm{sec}$ earlier and more rapidly than that at $50 \mathrm{cycle} / \mathrm{sec}$, just as seen experimentally. Therefore, the membrane resistance of neuroglia increases during $\mathrm{SD}$. (Since the $5 \mathrm{cycle} / \mathrm{sec}$ frequency line on Fig. 3 would be between the line through $\tau \omega=0.3$ on $2 \lambda / b=1$ and the real axis, no measurable phase angle would be expected at $5 \mathrm{cycle} / \mathrm{sec}$ regardless of the membrane resistance of neurons or neuroglia.)

It is not possible to do much to quantitate this increase in neuroglia membrane resistance, for it does not seem possible to distinguish quantitative changes in membrane resistance of neuroglia from that of neurons after approximately $15 \mathrm{sec}$ of SD. However, the phase angle at $500 \mathrm{cycle} / \mathrm{sec}$ always starts to decrease while the phase angle at $50 \mathrm{cycle} /$ $\mathrm{sec}$ is still at its maximum. Therefore the membrane resistance of neuroglia must be already decreasing at the time the phase angle at $50 \mathrm{cycle} /$ sec is at its maximum and the membrane resistance of neurons must be increasing.

It does not seem possible to put any restrictions on the homogeneity 
of the changes in neuroglia. Indeed, since the neuroglial population is not homogeneous morphologically, it seems unlikely that it acts homogeneously during $\mathrm{SD}$.

\section{Discussion}

Two revisions must be made in the analysis of current flow in cortex. In explaining why current flow across cell bodies need not be considered, it was argued that the cell body could be considered as the midpoint of a cable and therefore should have little transmembrane current (19). However, this argument is only true for one-third of the dendrites, so there might be some phase angle changes at 5,000 cycle/sec due to cell bodies. Another point is the degree of applicability of the Maxwell (15) type approach for current flow normal to the axis of a fiber (transverse flow). It was argued that the condition of large interstitial space was met because current flow along the axis of a fiber (longitudinal) was effectively at infinite frequency at $5,000 \mathrm{cycle} / \mathrm{sec}$ from the point of view of the longitudinal component of the specific impedance. However, the longitudinal flow is still restricted to the direction of the fiber, so that the longitudinal current flow is not effectively through interstitial space when considered from the point of view of the Maxwell type solution for transverse current flow. The solution for transverse current flow with a small interstitial space is not known; however, the smaller the space the more current will flow through cells, so phase angles would be expected to open at lower frequencies than those predicted by the equations. This may be part of the explanation for the phase angle at 5,000 cycle/ $\mathrm{sec}$ in non-SD cortex.

There seems to be little doubt that the decrease in membrane resistance of neurons is due to depolarization and that the depolarization is due to the increased concentration of potassium in the interstitial space which occurs during $\operatorname{SD}(3,14,16)$. Since the changes in transmembrane potential caused by the testing current used in measuring specific impedance are small compared to the depolarizations associated with the membrane resistance change, the resistance actually measured is a slope resistance, not a chord resistance. Therefore the decrease of about 6.6 times can be compared to the decrease of 1.8 to 7 times observed by Araki and Terzuolo during depolarizations in "voltage clamped" cat motoneurons (2).

During SD this decreased membrane resistance in a depolarized area becomes a low resistance source and more effectively depolarizes areas at a distance on the same fiber. Assume that this decrease in membrane 
resistance is due to an increase in membrane permeability to potassium ("delayed rectification") and the Grafstein's (4) potassium depolarization theory of SD is true. Then during SD the neuronal membrane will be closer to the potassium equilibrium potential which is now on the depolarized side of the resting potential. Therefore the cell will be more depolarized than if delayed rectification had not occurred. Both these effects of the decreased membrane resistance of neurons will contribute to the "pseudothreshold" nature of SD. The decrease in size of the interstitial space might be expected to further increase the concentration of potassium in this space, perhaps contributing to the regenerative nature of SD.

If the usual assumption is made that chloride is in equilibrium across neuronal membranes, it then follows that when a cell is depolarized there will be a force driving chloride into the cell. Van Harreveld $(20,21)$ has presented evidence that there is a net motion of chloride from interstitial space in SD. The similarity in time course of decrease of neuronal membrane resistance and decrease in size of the interstitial space gives additional support to the connection between the two.

The finding of Rall and Patlack (17) of a decrease of "inulin space" in white matter overlying perfused ventricle after death, presumably with depolarization of cells, would appear to be related to a similar mechanism. Similar reasoning holds for the increased impedance, presumably due to decreased interstitial space, during asphyxia and circulatory arrest $(21,22)$. However, since the forces maintaining the normal separation of about $100 \AA$ between cells in cortex are not understood, it seems best to reserve judgment on this issue.

The range of acceptable size of the "equivalent conductive interstitial space" of from 10 to $15 \%$ of cortex begins to be consistent with many of the "large interstitial space" views $(14,16,22)$. However, it is also consistent with a major conclusion from electron microscopy-that the extracellular space is in narrow sheets between cells (8). In fact, there is recent electron-microscopical evidence that the space between cells is an aqueous space (12). Changes in the size of the interstitial space of brain may occur during fixation for electron microscopy. However, the view of the central nervous system derived from the results of electron microscopy such as that developed by Horstmann and Meves (8) and used in this impedance work would still hold.

The increased membrane resistance of neuroglia does not seem to have been reported or even suggested before. Hild and co-workers (6) 
have shown depolarization of astrocytes when external potassium concentration is increased, which suggests that the increased membrane resistance of neuroglia is associated with depolarization. The prolonged depolarizations of astrocytes in tissue culture initiated by passing currents from extracellular electrodes, largely independent of the direction of current flow (6), may well have a similar basis. if astrocytes have higher membrane resistance to outward depolarizing current than to inward current, the net effect of current passed from electrodes outside the cell will be a depolarization of the cell body which is in the middle of the cell, regardless of direction of current flow outside the cell. The cell will then repolarize with the time constant of the membrane. ${ }^{3}$ Hild and Tasaki (7) have found the current voltage relations in glial cells to be approximately linear up to changes of about $20 \mathrm{mv}$. The effect of larger changes in voltage is not known.

Since astrocytes depolarize when the external potassium concentration is increased, the permeability of astrocytes to potassium is at least of the same order as that of the other common ionic species. This suggests that the increased membrane resistance of neuroglia in SD is at least partially a decrease in potassium conductance associated with a depolarization due to increased external potassium concentration. However, this is just the phenomenon of anomalous rectification, well known in skeletal muscle (1). It is currently unclear whether anomalous rectification is a function of the absolute value of membrane potential or of the difference in membrane potential from the equilibrium potential of potassium (i.e., a function of the driving force on potassium). The fact that the increased membrane resistance of neuroglia occurs so late in the course of SD (at a time when the concentration of potassium in the interstitial space may well be decreasing) would seem to favor the driving force on potassium as the most likely determinant of potassium permeability in the case of neuroglia.

If the rectification of ncuroglia is due to a decreased potassium permeability under an outward driving force, then potassium will enter neuroglia more readily than it leaves and neuroglia will help keep the interstitial potassium concentration low. There is suggestive evidence that neuroglia can bind potassium $(10,11)$. This mechanism would also "buffer" the interstitial space against rises in potassium concentration. Neurons in the hippocampus lie next to each other without interposed neuroglia (5).

3 Dr. J. W. Woodbury suggested this interpretation. 
There is indirect evidence of potassium accumulation in the interstitial space in the hippocampus when its neurons fire (9). This suggests that this potassium accumulation in hippocampus is due to a lack of neuroglia to "suck up" the potassium. Assuming Grafstein's (4) potassium depolarization theory of SD is correct, then SD may be a case of saturating the ability of neuroglia to take up potassium. The success of pretreatment of cortex with high potassium solutions in facilitating the production of SD (14) is consistent with this interpretation.

\section{References}

1. Adrian, R. H., and W. H. Freygang. 1962. The potassium and chloride conductance of frog muscle membrane. J. Physiol. London 163: 61-103.

2. ARAKI, T., and C. A. Terzuolo. 1962. Membrane currents in spinal motoneurons associated with the action potential and synaptic activity. $J$. Neurophysiol. 25: 772-789.

3. Brinlex, F. J., JR., E. R. Kandel, and W. H. Marshall. 1960. Potassium outflux from rabbit cortex during spreading depression. J. Neurophysiol. 23: 246-256.

4. Grafstein, B. 1956. Mechanism of spreading cortical depression. J. Neurophysiol. 19: 154-171.

5. Green, J. D., and D. S. Maxwell. 1961. Hippocampal electrical activity I. Morphological aspects. Electroencephalog. Clin. Neurophysiol. 13: 837-846.

6. Hild, W., J. J. Chang, and I. Tasaki. 1958. Electrical responses of astrocylic glia from the mammalian central nervous system cultivated in vitro. Experientia 14: $220-221$.

7. HiLd, W., and I. Tasaki. 1962. Morphological and physiological properties of neurons and glial cells in tissue culture. J. Neurophysiol. 25: 277-304.

8. Horstmann, E., and H. Meves. 1959. Die Feinstruktur des molekularen Rindengranes und ihre physiologische Bedeutung. Z. Zellforsch. 49: 569-604.

9. Kandel, E. R., and W. A. SpEncer. 1961. Electrophysiology of hippocampal neurons. II. After-potentials and repetitive firing. J. Neurophysiol. 24: 243-259.

10. Katzman, R., and P. H. Lemerman. 1953. Brain potassium exchange in normal adult and immature rats. Am. J. Physiol. 175: 263-270.

11. Koch, A., J. B. Ranck, JR., and B. L. Newman. 1962. Ionic content of the neuroglia. Exptl. Neurol. 6: 186-200.

12. Lasansky, A., and F. Wald, 1962. The extra cellular space in the toad retina as defined by the distribution of ferrocyanide. A light and electron microscope study. J. Cell Biol. 15: 463-481.

13. Leao, A. A. P., and Martins Ferreira, 1953. Alteracao da impedancia electrica no decurso da depresso abastrante da atvidade do cortex cerebral. Anais Acad. Brasil. Cienc. 25: 259-266. [Translation obtained from NIH Library.]

14. Marshald, W. H. 1959. Spreading cortical depression of Lẽoa. Physiol. Rev. 39: $239-279$.

15. Maxwell, J. C. 1891. "A Treatise on Electricity and Magnetism," 3rd ed, Sect. 310-314. Dover, New York. 
16. Ochs, S. 1962. The nature of spreading depression in neural-networks. Intern. Rev. Neurobiol. 4: 1-69.

17. Rall, D. P., and Patlak, C. S. 1962. The extra cellular space in brain. Federation Proc. 21: 324.

18. Ranck, J. B., JR., 1963a. Specific impedance of rabbit ccrebral cortex. Exptl. Neurol. 7: 144-152.

19. RaNCK, J. B., JR., 1963. Analysis of specific impedance of rabbit cerebral cortex. Exptl. Neurol. 7: 153-174.

20. Van Harreveid, A. 1958. Changes in the diameter of apical dendrites during spreading depression. Am. J. Physiol. 192: 457-463.

21. Van Harreveld, A., and J. P. Schade, 1959. Chloride movements in cerebral cortex after circulatory arrest and during spreading depression. J. Cell. Comp. Physiol. 54: 65-84.

22. Van Harreveld, A., and J. P. Schade, 1960. On the distribution and movements of water and electrolytes in the cerebral cortex, pp. 239-256. In "Structure and Function of the Cerebral Cortex." D. B. Tower and J. P. Schade [eds.]. Elsevier, New York. 